\title{
Perception and Pain Thresholds of tDCS and tACS
}

\author{
E. KVAŠŇÁKK ${ }^{1}$ \\ ${ }^{1}$ Department of Medical Biophysics and Medical Informatics, Third Faculty of Medicine, Charles \\ University, Prague, Czech Republic
}

Received August 23, 2019

Accepted November 11, 2019

\begin{abstract}
Summary
Transcranial electric stimulation (tES) protocols strictly need to keep the safety of stimulations and to avoid the unpleasant feelings of stimulated subjects. Following that, the aim of the paper was to find out both the perception and the pain thresholds of different tES modalities. The perception thresholds and the pain thresholds at AF7 and AF8 electrodes with transcranial direct and alternating current stimulation (tDCS and tACS) were performed. Average perception and pain thresholds did not differ significantly inter-individually but intra-individually. With gradually increasing intensity the analysis of thresholds showed that 1) an average participant perceived as first $20 \mathrm{~Hz}$ and $40 \mathrm{~Hz}$ tACS, then $10 \mathrm{~Hz}$ tACS and cathodal tDCS and then anodal tDCS, and 2) the pain was felt first for anodal tDCS, then for 6,10 and $20 \mathrm{~Hz}$ tACS, then for cathodal tDCS and finally for $40 \mathrm{~Hz}$ tACS. Besides that, intensity-dependent regularities in feelings of different tES modalities were found. Perception thresholds for 2 types of tDCS (anodal and cathodal) and 4 types of tACS (6-40 Hz) are arranged in sequence of gradually increased values (threshold current): anodal tDCS - cathodal tDCS $-6-10-20-40 \mathrm{~Hz}$ tACS. whereas pain thresholds are arranged with decreased threshold values.
\end{abstract}

\section{Key words}

Perception • Pain • Threshold • Transcranial electrical stimulation

\section{Corresponding author}

E. Kvašnák, Department of Medical Biophysics and Medical Informatics, Third Faculty of Medicine, Charles University, Prague, Czech Republic. E-mail: eugen.kvasnak@lf3.cuni.cz

\section{Introduction}

Transcranial electrical stimulation with direct and alternating current (tDCS and tACS) are non-invasive methods used in experiments focused on therapeutic purposes (e.g. pain treatment, neurological and psychiatric diseases, etc.) as well as cognitive effects (working memory, attention, language skills, numerical skills etc.). Since the first reports of tDCS (Lippold and Redfearn 1964, Priori et al. 1998, Nitsche and Paulus 2000 and 2001, Nitsche et al. 2003) and similarly tACS (Antal et al. 2008, Antal and Herrmann 2016) there are ongoing efforts of tuning-up the stimulation protocols, including thresholds of both the perception and the pain, as for clinical therapy as for research. In both, tDCS and tACS, are studies (Nitsche et al. 2003, Iyer et al. 2005, Lagopoulos and Degabriele 2008, Bikson et al. 2009, Dell'Osso et al. 2011, Brunoni et al. 2011, Antal et al. 2016) reporting also some adverse effects which are usually mild and disappear soon after stimulation. Preparation of all kinds of application protocols follows, besides desired stimulation effects, also the aim of keeping safety and avoiding unpleasant feelings caused by electric stimulation. Thus, there is a need of knowing the thresholds of the perception and pain as well as the relationship among threshold values for individual participant.

\section{Methods}

Experiments were performed with 60 healthy participants (age 18-23, average age 19) without both the history of epilepsy and the wounds on frontal skull area. Participants were exposed to stimulation set which consists from random order of anodal tDCS, cathodal tDCS, $6 \mathrm{~Hz}$ tACS, $10 \mathrm{~Hz}$ tACS, $20 \mathrm{~Hz}$ tACS and $40 \mathrm{~Hz}$ tACS. Stimuli were generated by Starstim (Neuroelectrics Ltd, Spain) with intensity from range $0.2-2.0 \mathrm{~mA}$ (increment $0.1 \mathrm{~mA}$ ) via circular sponge electrodes 
(diameter $5 \mathrm{~cm}$ ) placed at AF7 and AF8 sites (10-20 system). Each stimulus lasted $10 \mathrm{sec}$ (RAMP $1 \mathrm{sec}$ ) followed by $6 \mathrm{sec}$ inter-stimuli pause. Participants were instructed to write down both perception threshold and pain threshold. The experiments were approved by the Ethics Committee of Third Faculty of Medicine, Charles University in Prague and they have been carried out in accordance with The Code of Ethics of the World Medical Association (Declaration of Helsinki) for experiments involving humans.

\section{Results}

\section{Perception}

According to the participant's reporting, the average perception thresholds did not differ significantly (Table 1). The minimum threshold of perception for all 6 kinds of stimulations (anodal-tDCS, cathodal-tDCS, tACS on 6, 10, 20 and $40 \mathrm{~Hz}$ ) was in average $0.2 \mathrm{~mA}$, whereas the maximum perception thresholds ranged from
0.13 for cathodal tDCS and $40 \mathrm{~Hz}$ tACS, to $0.17 \mathrm{~mA}$ for anodal tDCS and $20 \mathrm{~Hz}$ tACS.

Although the average thresholds for 6 different simulations did not differ significantly, the regularities in intra-individual relationships of perception thresholds of different stimulation modalities were found (Fig. 1). Based on intra-individual comparison of thresholds for different stimulations, the perception threshold for anodal tDCS and $6 \mathrm{~Hz}$ tACS were usually the same and higher than thresholds for cathodal tDCS and for $10 \mathrm{~Hz}$ tDCS by $15 \%$. Thresholds for cathodal tDCS and for $10 \mathrm{~Hz}$ tDCS were higher than thresholds for $20 \mathrm{~Hz}$ tACS by $26 \%$. Threshold for $20 \mathrm{~Hz}$ was usually higher than threshold for $40 \mathrm{~Hz}$ tACS by $5 \%$.

Describing perception thresholds of tES with gradually increasing intensity, we can say that an average participant sense first $40 \mathrm{~Hz}$ tACS, then $20 \mathrm{~Hz}$ tACS, then $10 \mathrm{~Hz}$ tACS together with cathodal tDCS and then the last (with highest threshold) anodal tDCS together with $6 \mathrm{~Hz}$ tACS.

Table 1: Average perception thresholds

\begin{tabular}{|c|c|c|c|c|c|}
\hline Stimulation & $\begin{array}{c}\text { Average } \\
\text { Threshold }\end{array}$ & STD & Minimum & Maximum & $\begin{array}{l}\text { Thresholds } \\
\quad<2 \mathrm{~mA}\end{array}$ \\
\hline Anodal tDCS & 0.60 & 0.34 & 0.20 & 1.70 & $95 \%$ \\
\hline Cathodal tDCS & 0.54 & 0.26 & 0.20 & 1.30 & $97 \%$ \\
\hline $6 \mathrm{~Hz} t A C S$ & 0.62 & 0.37 & 0.20 & 1.60 & $98 \%$ \\
\hline $10 \mathrm{~Hz} t A C S$ & 0.57 & 0.30 & 0.20 & 1.60 & $98 \%$ \\
\hline $20 \mathrm{~Hz} t A C S$ & 0.53 & 0.34 & 0.20 & 1.70 & $98 \%$ \\
\hline $40 \mathrm{~Hz} t A C S$ & 0.56 & 0.29 & 0.20 & 1.30 & $97 \%$ \\
\hline
\end{tabular}

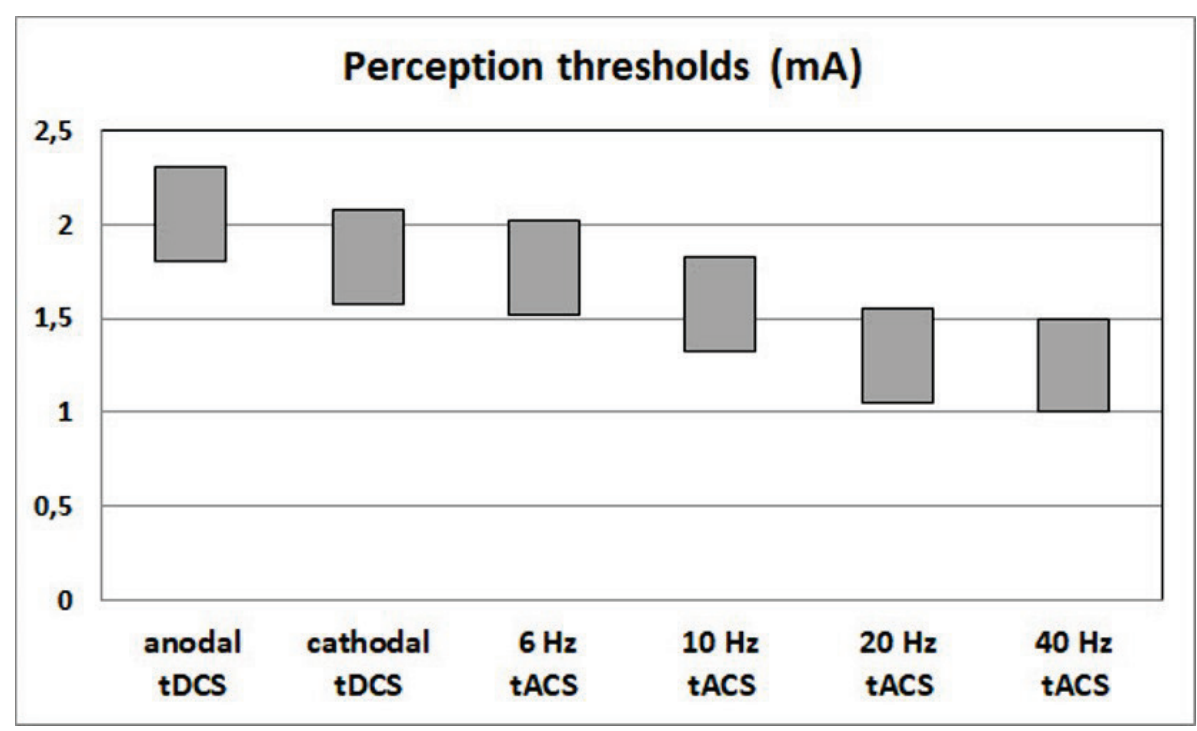

Fig. 1. Relative relationships among perception thresholds of different transcranial electrical stimulation modalities. 
Pain

Average pain thresholds for stimuli we applied did now differ significantly (Table 2). For anodal tDCS was the average pain threshold $1.14 \mathrm{~mA}$, for cathodal tDCS $1.34 \mathrm{~mA}$, for $6 \mathrm{~Hz}, 10 \mathrm{~Hz}$ and $20 \mathrm{~Hz}$ tACS threshold was 1.39 whereas for 40 tACS the average pain threshold was $1.52 \mathrm{~mA}$. The minimum pain threshold in average was $0.30 \mathrm{~mA}$ for anodal tDCS and 0.40 for other 5 kinds of stimulations. For each stimulation modality, the pain threshold was higher than $2.00 \mathrm{~mA}$ for half of participants (Table 2).

Analysis of intra-individual relationships among the pain threshold of different stimulations showed that the situation is the opposite of that for perception thresholds (Fig. 2). Individual pain threshold for anodal tDCS was usually lower than pain threshold for cathodal tDCS by $23 \%$. Threshold for $6 \mathrm{~Hz}$ tACS was higher than threshold for cathodal tDCS by $11 \%$. Threshold for $10 \mathrm{~Hz}$ tACS was higher than threshold for $6 \mathrm{~Hz}$ tACS by $9 \%$. Threshold for $20 \mathrm{~Hz}$ tACS was higher than threshold for $10 \mathrm{~Hz}$ tACS by $9 \%$. Threshold for $40 \mathrm{~Hz}$ tACS was higher than threshold for $20 \mathrm{~Hz}$ tACS by $17 \%$.

Pain thresholds (for an average participant) of different tES modalities were found in following sequence: pain was first felt for anodal tDCS, then for cathodal tDCS, then for $6 \mathrm{~Hz}$, then for $10 \mathrm{~Hz}$, then for $20 \mathrm{~Hz}$ tACS and then for $40 \mathrm{~Hz}$ tACS.

Table 2. Average pain thresholds

\begin{tabular}{|c|c|c|c|c|c|}
\hline Stimulation & $\begin{array}{l}\text { Average } \\
\text { Threshold }\end{array}$ & STD & Minimum & Maximum & $\begin{array}{c}\text { Thresholds } \\
<2 \mathrm{~mA}\end{array}$ \\
\hline Anodal tDCS & 1.14 & 0.45 & 0.30 & 2.00 & $47 \%$ \\
\hline Cathodal tDCS & 1.34 & 0.50 & 0.40 & 2.00 & $57 \%$ \\
\hline $6 \mathrm{~Hz} t A C S$ & 1.39 & 0.51 & 0.40 & 2.00 & $47 \%$ \\
\hline $10 \mathrm{~Hz} t A C S$ & 1.40 & 0.45 & 0.40 & 2.00 & $52 \%$ \\
\hline $20 \mathrm{~Hz} t A C S$ & 1.39 & 0.48 & 0.40 & 2.00 & $57 \%$ \\
\hline $40 \mathrm{~Hz} t A C S$ & 1.52 & 0.46 & 0.40 & 2.00 & $55 \%$ \\
\hline
\end{tabular}

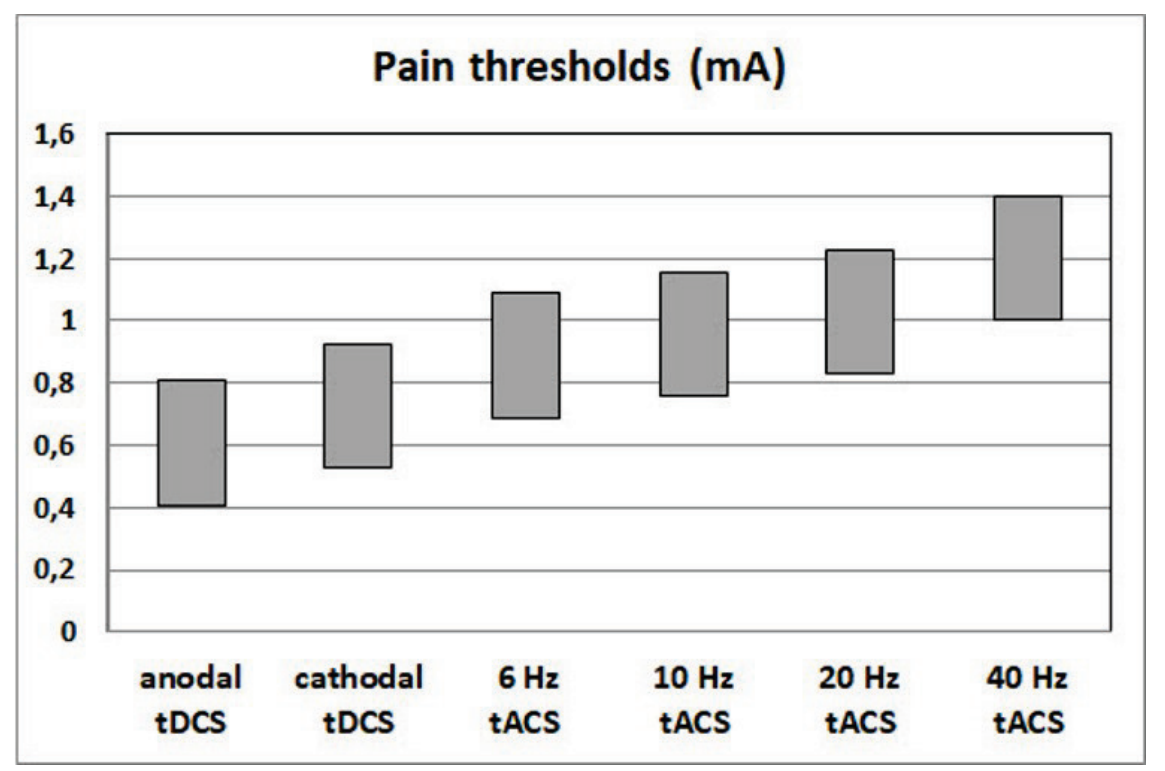

Fig. 2. Relative relationships among pain thresholds of different transcranial electrical stimulation modalities.

\section{Discussion}

Our experimental results showed that the perception and the pain threshold for anodal tDCS, cathodal tDCS and tACS on 4 different frequencies $(6 \mathrm{~Hz}, 10 \mathrm{~Hz}, 20 \mathrm{~Hz}$ and $40 \mathrm{~Hz}$ ) did not differ in average significantly. On the other hand, they differed interindividually. This phenomenon can be plausible 
explained by different individual sensitivity to transcranial stimulation as described elsewhere. Most important outcome of our results is general relationship among intra-individual thresholds for different types of stimulations.

Different perception and discomfort (sometimes referred as a pain) during transcutaneous electrical stimulation could be explained by the excitation of peripheral nerves; electrochemical processes (Minhas et al. 2011), facilitated by sweat, skin wounds etc. (Nitsche et al. 2003, Datta et al. 2008). Our finding of adverse effects occurrence corresponds with some other studies (Brunoni et al. 2011, Minhas et al. 2011) reporting about tingling, headache, discomfort and burning perception but should be pointed out our study did not evaluate after effects of electrical stimulation unlike some other studies (Nitsche et al. 2008, Palm et al. 2008, Palm et al. 2008, Fertonani et al. 2015). Like some studies (Turi et al. 2013, Ambrus et al. 2010, Raco et al. 2014) our healthy subjects felt often cutaneous perceptions during tACS; we did not record any dizziness, probably because of very short stimulation time. On the other hand, some studies (Chaieb et al. 2014, Naro et al. 2016) stimulating frontally with $2 \mathrm{~mA}$ for $5 \mathrm{~min}$ tACS at 10, 50, 300, $50000 \mathrm{~Hz}$ found no adverse events at all. Congruently with our findings, all reported adverse events of tACS have been transient rather than persistent (Raco et al. 2014, Naro et al. 2016). Summarizing, whereas in average the perception and pain thresholds of tDCS and tACS at 6, 10, 20 and $40 \mathrm{Hs}$ seems to be equal, there are intra-individual regularities among thresholds for 6 different stimulation modalities.

As regarding sensitivity to different transcranial electrical stimulation types, we report here, the average participant sensed at lowest intensity 20 and $40 \mathrm{~Hz}$ tACS then cathodal tDCS, and then on $20 \%$ lower intensities than anodal tDCS or $6 \mathrm{~Hz}$ tACS. Participants usually started to sense $20 \mathrm{~Hz}$ or $40 \mathrm{~Hz}$ tACS at intensities by 20-28\% lower than they sensed $10 \mathrm{~Hz}$ tACS. Average participant started to perceive $6 \mathrm{~Hz}$ tACS at intensity $22 \%$ higher than he/she started to perceive $10 \mathrm{~Hz}$ tACS and $50 \%$ higher than he/she started to perceive $20 \mathrm{~Hz}$ tACS.

From intra-individual relationships point of view, the relationships between pain thresholds values for different types of stimulation are the opposite of those for perception thresholds values (Table 4). Individual pain threshold for anodal tDCS was usually lower than pain threshold for cathodal tDCS by $28 \%$ and lower than threshold for $6 \mathrm{~Hz}$ tACS by $18 \%$. Individual pain thresholds for $6 \mathrm{~Hz}, 10 \mathrm{~Hz}$ and $20 \mathrm{~Hz}$ did not differ significantly. Individual threshold for $40 \mathrm{~Hz}$ was usually higher by $14-20 \%$ than for three other tACS stimulations.

\section{Conflict of Interest}

There is no conflict of interest.

\section{Acknowledgements}

This work was supported by Czech Health Research Council (grant \# AZV 15-31538A).

\section{References}

AMBRUS CG, PAULUS W, ANTAL A: Cutaneous perception thresholds of electrical stimulation methods: comparison of tDCS and tRNS. Clin Neurophysiol 121: 1908-1914, 2010.

ANTAL A, HERRMANN CS: Transcranial alternating current and random noise stimulation: possible mechanisms. Neural Plast 2016: 3616807, 2016.

ANTAL A, BOROS K, POREISZ C, CHAIEB L, TERNEY D, PAULUS W: Comparatively weak after-effects of transcranial alternating current stimulation (tACS) on cortical excitability in humans. Brain Stimul 1: 97-105, 2008.

BIKSON M, DATTA A, ELWASSIF M: Establishing safety limits for transcranial direct current stimulation. Clin Neurophysiol 120: 1033-1034, 2009.

BRUNONI AR, AMADERA J, BERBEL B, VOLZ MS, RIZZERIO BG, FREGNI F: A systematic review on reporting and assessment of adverse effects associated with transcranial direct current stimulation. Int $J$ Neuropsychopharmacol 14: 1133-1145, 2011.

BRUNONI AR, VALIENGO L, ZANAO T, D OLIVEIRA JF, BENSENOR IM, FREGNI F: Manic psychosis after sertraline and transcranial direct-current stimulation. J Neuropsychiatry Clin Neurosci 23: E4-E5, 2011.

CHAIEB L, ANTAL A, PAULUS W: Transcranial alternating current stimulation in the low kHz range increases motor cortex excitability. Restor Neurol Neurosci 29: 167-175, 2011. 
CHAIEB L, ANTAL A, PISONI A, SAIOTE C, OPITZ A, AMBRUS GG: Safety of $5 \mathrm{kHz}$ tACS. Brain Stimul 7: 92-96, 2014.

DATTA A, ELWASSIF M, BATTAGLIA F, BIKSON M: Transcranial current stimulation focality using disc and ring electrode configurations: FEM analysis. J Neural Eng 5: 163-174, 2008.

DELL'OSSO A, PRIORI A, ALTAMURA AC: Efficacy and safety of transcranial direct current stimulation in major depression. Biol Psychiatry 69: e23-e24, 2011.

FERTONANI A, FERRARI C, MINIUSSI C: What do you feel if I apply transcranial electric stimulation? Safety, perception s and secondary induced effects. Clin Neurophysiol 126: 2181-2188, 2015.

IYER MB, MATTU U, GRAFMAN J, LOMAREV M, SATO S, WASSERMANN EM: Safety and cognitive effect of frontal DC brain polarization in healthy individuals. Neurology 64: 872-875, 2005.

LAGOPOULOS J, DEGABRIELE R: Feeling the heat: the electrode-skin interface during DCS. Acta Neuropsychiatrica 20: 98-100, 2008.

LIPPOLD OC, REDFEARN JW: Mental changes resulting from the passage of small direct currents through the human brain. Br J Psychiatry 110: 768-772, 1964.

MINHAS P, DATTA A, BIKSON M: Cutaneous perception during tDCS: role of electrode shape and sponge salinity. Clin Neurophysiol 122/4: 637-638, 2011.

MOLIADZE V, ANTAL A, PAULUS W: Boosting brain excitability by transcranial high frequency stimulation in the ripple range. $J$ Physiol 588: 4891-904, 2010.

NARO A, LEO A, RUSSO M, CANNAVO A, MILARDI D, BRAMANTI P, CALABRO RS: Does transcranial alternating current stimulation induce cerebellum plasticity? Feasibility, safety and efficacy of a novel electrophysiological approach. Brain Stimul 9: 388-395, 2016.

NITSCHE MA, COHEN LG, WASSERMAN EM, PRIORI A, LANG N, ANTAL A, PAULUS W, HUMMEL F, BOGGIO PS, FREGNI F, PASCUAL-LEONE A: Transcranial direct current stimulation: State of the art. Brain Stimul 1: 206-223, 2008.

NITSCHE MA, PAULUS W: Excitability changes induced in the human motor cortex by weak transcranial direct current stimulation. J Physiol 527: 633-639, 2000.

NITSCHE MA, PAULUS W: Sustained excitability elevations induced by transcranial DC motor cortex stimulation in humans. Neurology 57: 1899-1901, 2001.

NITSCHE MA, LIEBETANZ D, LANG N, ANTAL A, TERGAU F, PAULUS W: Safety criteria for transcranial direct current stimulation (tDCS) in humans. Clin Neurophysiol 114: 2220-2222, 2003.

FRANK E, WILFURTH S, LANDGREBE M, EICHHAMMER P, HAJAK G, LANGGUTH B: Anodal skin lesions after treatment with transcranial direct current stimulation. Brain Stimu 3: 58-59, 2010.

PALM U, KEESER D, SCHILLER C, FINTESCU Z, NITSCHE M, REISINGER E, PADBERG F: Skin lesions after treatment with transcranial direct current stimulation (tDCS). Brain Stimul 1: 386-387, 2008.

PRIORI A, BERARDELLI A, RONA S, ACCORNERO N, MANFREDI M: Polarization of the human motor cortex through the scalp. NeuroReport 9: 2257-2260, 1998.

RACO V, BAUER R, OLENIK M, BRKIC D, GHARABAGHI A: Neurosensory effects of transcranial alternating current stimulation. Brain Stimul 7: 823-831, 2014.

TURI Z, AMBRUS GG, JANACSEK K, EMMERT K, HAHN L, PAULUS W, ANTAL A: Both the cutaneous perception and phosphene perception are modulated in a frequency-specific manner during transcranial alternating current stimulation. Restor Neurol Neurosci 31: 275-285, 2013. 